\title{
Validation of a quantitative image analysis methodology for the assessment of the morphology and structure of aerobic granular sludge in the presence of pharmaceutically active compounds
}

\author{
Cristiano Leal ${ }^{\mathrm{a}}$, Angeles Val del Río ${ }^{\mathrm{b}}$, Eugénio C. Ferreira ${ }^{\mathrm{a}}$, Daniela Mesquita ${ }^{\mathrm{a}}$, \\ António L. Amaral ${ }^{\mathrm{a}, \mathrm{c}, \mathrm{d}, *}$ \\ ${ }^{\text {a } C E B}$ - Centre of Biological Engineering, Universidade do Minho, Campus de Gualtar, 4710-057 Braga, Portugal \\ ${ }^{\mathrm{b}}$ CRETUS Institute, Department of Chemical Engineering, Universidade de Santiago de Compostela, E-15705, Santiago de \\ Compostela, Spain \\ c Instituto Politécnico de Coimbra, Instituto Superior de Engenharia de Coimbra, Rua Pedro Nunes, Quinta da \\ Nora, 3030-199 Coimbra, Portugal \\ d Instituto de Investigação Aplicada, Laboratório SiSus, Rua Pedro Nunes, Quinta da Nora, 3030-199 Coimbra, Portugal
}

\section{A R T I C L E I N F O}

\section{Article history:}

Received 8 December 2020

Received in revised form 7 April 2021

Accepted 16 May 2021

Available online 17 May 2021

\section{Keywords:}

Quantitative image analysis

Aerobic granular sludge

Structural descriptors

Pharmaceutically active compounds

Methodology validation

\begin{abstract}
A B S T R A C T
Aerobic granular sludge is considered a promising technology for biological wastewater treatment, since it is more compact and resistant to shock and toxic loads than activated sludge systems. Furthermore, the monitoring of the aerobic granules structure, size and morphology has been growing in importance, proving to be suitable for operational and process control purposes. In this work, a sampling methodology is validated for the assessment of the ensemble granular and floccular biomass, by quantitative image analysis, in the presence of pharmaceutically active compounds, including $17 \beta$-estradiol and $17 \alpha$-ethinylestradiol and the sulfamethoxazole. The employed sampling volumes were found to be adequate in obtaining a representative number of granules for all samples. Indeed, it was always obtained a variation lower than $5 \%$ in the average and standard deviation criterion of the main size, morphological and structural parameters. Moreover, it was also assessed the performance of the sieving process in successfully separating the granular and floccular biomass fractions and established a lower bound fraction size cut-off for the employed sieve. In addition, the continuity of the performed methodology and the image acquisition triplicates approach could also be validated. The ability of the proposed QIA procedure to monitor the studied AGS system was corroborated by the fact that $95 \%$ of the samples were able to be classified in the correct group (proof of concept).
\end{abstract}

(c) 2021 Elsevier B.V. All rights reserved.

\section{Contents}

1. Introduction 2

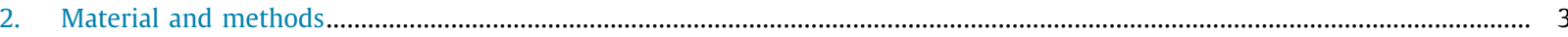

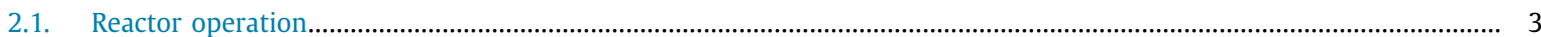

* Corresponding author at: Instituto Politécnico de Coimbra, Instituto Superior de Engenharia de Coimbra, Rua Pedro Nunes, Quinta da Nora, 3030-199 Coimbra, Portugal.

E-mail address: lpamaral@isec.pt (A.L. Amaral). 
2.2. Sludge sampling and image acquisition.

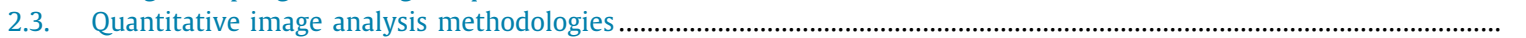

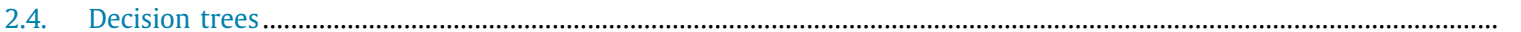

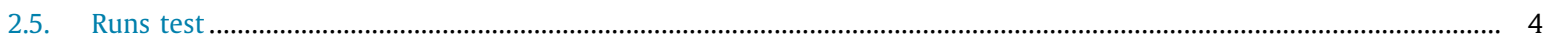

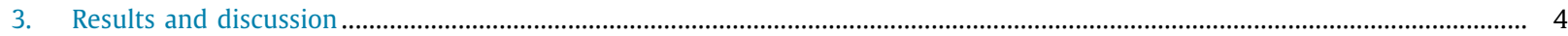

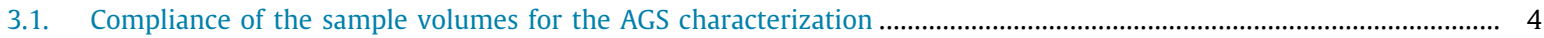

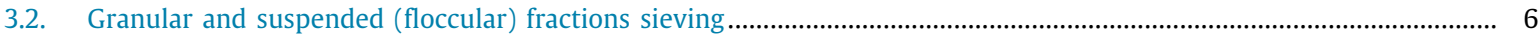

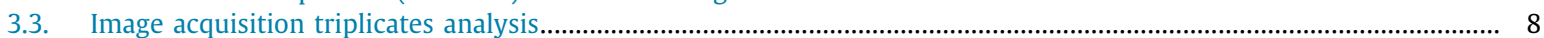

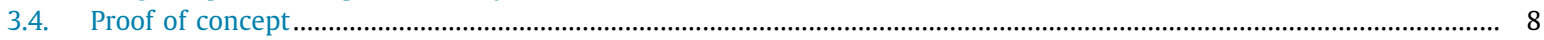

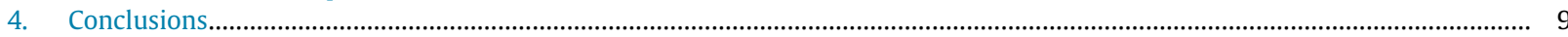

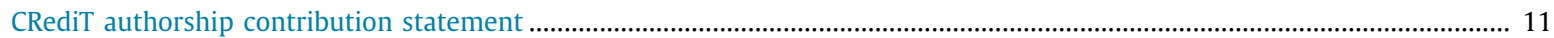

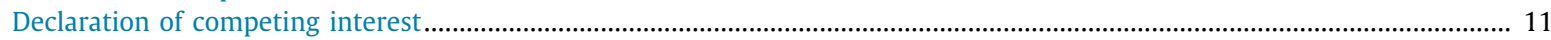

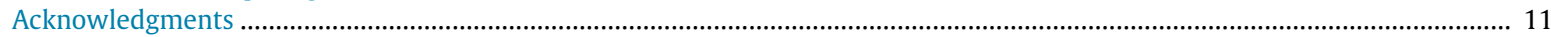

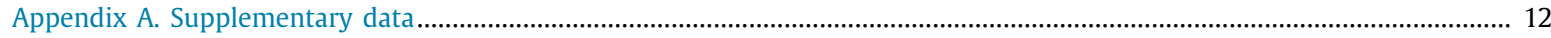

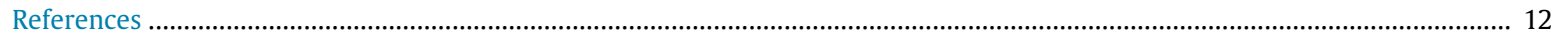

\section{Introduction}

Aerobic granular sludge (AGS) is considered a promising technology for wastewater treatment, being compact, cost-effective, highly resistant to toxic compounds and potentially capable to remove nutrients, toxic, and recalcitrant compounds. For that reason, AGS is now replacing activated sludge systems in several countries (Bengtsson et al., 2019; Maszenan et al., 2011; Nancharaiah and Kumar, 2017). As the obtained aggregates dimensions were found to be important for process control, granular size assessment of the aerobic granules is gaining increasing attention (Long et al., 2019; Verawaty et al., 2013; Zhang et al., 2015). Literature can be already found reporting the relevance of optimizing size granules distribution in such reactors (Zhou et al., 2016). Other studies revealed that a granule-sized based discharge could enhance the stability and control of AGS processes (Li et al., 2006; Sheng et al., 2010; Zhu et al., 2013). Also, it was previously found that the granules size can influence the performance of, particularly, phosphorous and nitrogen compounds' removal from wastewaters in a sequencing batch reactor (SBR) (Li et al., 2019). More recently, the granules size was found to be correlated with the content and composition of extracellular polymeric substances in AGS systems (Rusanowska et al., 2019) and relevant for membrane fouling monitoring in membrane bioreactors (Zhang and Jiang, 2019).

The stability of the AGS process is dependent on the balance between the floccular and granular fractions in the system (Aqeel et al., 2019). Moreover, several studies could be found in literature relating to sludge fractionation, including the use of different sieves size and different sludge volumes for fractions separation (Cheng et al., 2018; Jahn et al., 2019; Pronk et al., 2015). In this sense, the application of techniques for both flocs and granules specific morphological and structural assessment is of major interest. In fact, several methods in literature already report the assessment of AGS structure directed to granules: (i) freezing microtome sections; (ii) dissolved oxygen (DO) microelectrode (to assess microbial density in granules through DO profiles in the granules); (iii) confocal laser scanning microscopy (CLSM); (iv) ultra-high-field NMR; as well as for separated flocs and granules fractions; and (v) magnetic resonance imaging (MRI) (Kirkland et al., 2020; Li et al., 2014; Ranzinger et al., 2020). Quantitative image analysis (QIA) can be considered a less invasive technique when compared to DO microelectrode and freezing microtome sections methods. When compared with NMR and MRI, QIA is able to provide a more in-depth analysis of the aggregates structure and morphology.

In this sense, QIA is an objective, time and cost-effective methodology that has already been applied to monitor disturbances in anaerobic granular sludge (Costa et al., 2010). Recently, QIA coupled with chemometric analysis, was successfully used for mature and stable AGS systems' monitoring, evaluating both granular and suspended (floccular) biomass (Leal et al., 2020b). However, studies encompassing the validation of these techniques, in terms of floccular and granular continuity under transient conditions (after fractions separation by sieving), mainly in the presence of pharmaceutically active compounds (PhAC), are still unexplored.

Taking the above into consideration, the main objective of this work is to evaluate the representativeness of a QIA based methodology for the assessment of the size, morphology and structure of aerobic granules in the presence of the $17 \beta$-estradiol (E2) and $17 \alpha$-ethinylestradiol (EE2) steroid estrogens and the sulfamethoxazole (SMX) antibiotic. The studied PhAC were selected based on the increasing environmental and human health concern of steroid estrogens and sulfonamides (Leal et al., 2020a). It is known that one of major sources of E2, EE2, and SMX in surface (and ground) waters is related to the inefficient removal of these compounds by conventional biological wastewater treatment systems (including activated sludge). Thus, the assessment of the morphological and structural changes in floccular and granular AGS fractions in the presence of E2, EE2, and SMX can be considered of major interest. Four different experiments, including a mature granules experiment (CONT), were encompassed in order to validate the continuity, adequateness and robustness of the proposed methodology for a wide variety of granular and floccular aggregates separated with a $500 \mu \mathrm{m}$ mesh sieve. 


\section{Material and methods}

\subsection{Reactor operation}

A 5L SBR was operated with AGS for the treatment of a synthetic wastewater containing $5.168 \mathrm{~g} \mathrm{~L}^{-1}$ of $\mathrm{C}_{2} \mathrm{H}_{3} \mathrm{O}_{2} \mathrm{Na} \cdot 3 \mathrm{H}_{2} \mathrm{O}$; $0.887 \mathrm{~g} \mathrm{~L}^{-1}$ of $\mathrm{MgSO}_{4} \cdot 7 \mathrm{H}_{2} \mathrm{O} ; 0.35 \mathrm{~g} \mathrm{~L}^{-1}$ of $\mathrm{KCl} ; 0.596 \mathrm{~g} \mathrm{~L}^{-1}$ of $\mathrm{Na}_{2} \mathrm{HPO}_{4} ; 0.286 \mathrm{~g} \mathrm{~L}^{-1}$ of $\mathrm{KH}_{2} \mathrm{PO}_{4} ; 1.894 \mathrm{~g} \mathrm{~L}^{-1}$ of NH $\mathrm{NH}_{4} \mathrm{Cl}$. A volume of $10 \mathrm{~mL} \mathrm{~L}^{-1}$ of a trace elements solution was added containing $1.5 \mathrm{~g} \mathrm{~L}^{-1}$ of FeCl $3.6 \mathrm{H}_{2} \mathrm{O} ; 0.15 \mathrm{~g} \mathrm{~L}^{-1}$ of H$_{3} \mathrm{BO}_{3} ; 0.03$ $\mathrm{g} \mathrm{L}^{-1}$ of $\mathrm{CuSO}_{4} \cdot 5 \mathrm{H}_{2} \mathrm{O} ; 0.18 \mathrm{~g} \mathrm{~L}^{-1}$ of $\mathrm{KI} ; 0.12 \mathrm{~g} \mathrm{~L}^{-1}$ of $\mathrm{MnCl}_{2} \cdot 4 \mathrm{H}_{2} \mathrm{O} ; 0.06 \mathrm{~g} \mathrm{~L}^{-1}$ of $\mathrm{Na}_{2} \mathrm{MoO}_{2} \mathrm{H}_{2} \mathrm{O} ; 0.12 \mathrm{~g} \mathrm{~L}^{-1}$ of $\mathrm{ZnSO}_{4} \cdot 7 \mathrm{H}_{2} \mathrm{O}$, and $0.15 \mathrm{~g} \mathrm{~L}^{-1}$ of $\mathrm{CoCL}_{2} \cdot 6 \mathrm{H}_{2} \mathrm{O}$ (adapted from (De Kreuk et al., 2005). Moreover, $2 \mathrm{mg} \mathrm{L}^{-1}$ of each studied PhAC was also fed into the reactor, alongside the high strength synthetic wastewater, for the corresponding experiment to mimic pharmaceutical industrial wastewater.

In order to characterize the mature granules, a prior experiment was conducted for 49 days in the absence of $\mathrm{PhAC}$, acting as a control (CONT). Furthermore, the inoculated biomass was set to acclimate for a period of 86 days before the E2 experiment data collection and 28 days before the EE2 and the SMX experiments. Each operational cycle in the SBR lasted for $6 \mathrm{~h}$ encompassing $120 \mathrm{~min}$ of feeding, $232 \mathrm{~min}$ of aeration, $3 \mathrm{~min}$ of settling and $5 \mathrm{~min}$ of withdrawal, with a hydraulic retention time of $12 \mathrm{~h}$. Care was taken to allow the AGS attaining somewhat similar characteristics, mainly in terms of the overall removal performance and granular biomass (fraction and large granules size), in the beginning of the monitoring period for all experiments (CONT, E2, EE2, and SMX). For that purpose, the AGS was allowed to recover to the initial steady-state conditions, between experiments, for a period of roughly one month without any PhAC addition. The SBR was operated at room temperature $\left(18-23^{\circ} \mathrm{C}\right)$, with an air flow of $7.50 \mathrm{~L} \mathrm{~min}^{-1}$, resulting in a superficial air velocity kept above $1.8 \mathrm{~cm} \mathrm{~s}^{-1}$ allowing to keep the granules mixture in suspension during the monitoring period. The experimental setup, encompassing the sludge sampling methodology can be found in the supplementary material.

\subsection{Sludge sampling and image acquisition}

It is known that the AGS collected from within the reactor is not exempt of representativeness issues regarding its structural analysis, particularly at laboratory scale. With the aim of minimizing this problem the following sampling methodology was proposed. A sludge volume of $600 \mathrm{~mL}$ was collected at mid-point depth in the reactor, in the beginning of the aeration stage, at regular time intervals, to obtain homogeneous and representative biomass samples. Furthermore, the sludge bed volume per sample volume (SBD/SV) on the collected samples was also surveyed in order to be proportional to the SBD/SV on the reactor, with the sampling procedure being repeated otherwise (Pronk et al., 2014). These samples were kept under low agitation conditions to avoid settling and promote the mixture between the solid and liquid phases, on one hand, and to avoid changes in the sludge morphology and structure by high shear stress conditions, on the other. Such a high sampling volume ( $12 \%$ of the reactor volume) was collected to avoid representativeness issues. Furthermore, the aliquots were collected with a micropipette with a sectioned tip (allowing larger aggregates to flow) for the separation of fractions prior to the QIA procedure, and the remaining volume was reintroduced in the reactor.

The separation of the granular and suspended (floccular) mature AGS was obtained using a $500 \mu \mathrm{m}$ sieve, allowing for the floccular aggregates to pass through, and with the retained granules carefully picked up by rinsing with distilled water (Leal et al., 2020b) to avoid physical disruption. Care was also taken to avoid the formation of a filtration cake by carefully pouring the sampled volume throughout the available filtration area. This procedure was employed with the aim of minimizing possible alterations in the biomass morphology and structure during the physical handling. The granular fraction total suspended solids (TSS) were determined according to standard methods (APHA, 1998).

Aliquots of a standardized volume were employed for the granules QIA analysis, based on the TSS of the granular fraction. For samples with TSS lower than $15 \mathrm{~g} \mathrm{~L}^{-1}$, a $35 \mathrm{~mL}$ sample aliquot was used, whereas for TSS higher than $15 \mathrm{~g}$ $\mathrm{L}^{-1}$, a $10 \mathrm{~mL}$ aliquot was employed, in order to avoid significantly incrementing the time spent on image acquisition. The separated granular biomass fraction was further deposited in a Petri dish. Images from the entire set of granules present in each aliquot volume were acquired using an Olympus SZ 40 stereomicroscope (Olympus, Shinjuku, Japan) under a total magnification of $15 \times$. For the particular case of the SMX experiment, a Leica S8AP0 stereomicroscope was used under a total magnification of $16 \times$. The number of acquired granules averaged from 840 ( \pm 380 ) for the $35 \mathrm{~mL}$ aliquots to 394 $( \pm 104)$ for the $10 \mathrm{~mL}$ aliquots.

Regarding the suspended (floccular) biomass, aliquots of $10 \mu \mathrm{L}$ (in triplicate) were collected with a micropipette, with a sectioned tip to allow larger aggregates to flow through, deposited onto a slide and let to air-dry. Images were further acquired in bright field microscopy with an Olympus BX51 microscope (Olympus, Shinjuku, Japan) or a Nikon Eclipse Ci-L (Nikon, Minato, Japan) under a total magnification of $40 \times$ for the CONT and E2 experiments and $100 \times$ for the EE2 and SMX experiments, resulting in a total of 150 images (average of 11,200 flocs) per sample.

\subsection{Quantitative image analysis methodologies}

The employed QIA methodology was based on the identification and characterization of the aerobic granular and floccular biomass using the previously developed routines, run on Matlab 7.8.0 (The Mathworks, Natick, MA). A more detailed description of the employed QIA methodology can be found in (Amaral, 2003). 
The QIA routine for the granules identification first performs a background correction (with or without the use of a previously acquired background image) to correct for non-uniform light differences. A contrast enhancement step is next performed, based on a Canny edge detection algorithm, to sharpen the granules edges and facilitate border recognition. Granules segmentation is then achieved based on a thresholding algorithm, to separate them from the background. Debris elimination is the subsequent step and is based mainly in a size morphological opening operation. The minimum equivalent diameter for a granule not to be considered debris, or floccular material trapped in the sieve, was set to $175 \mu \mathrm{m}$, considering the limitations posed by the higher magnification used for the granules image acquisition, which did not allow to accurately identify and characterize aggregates below this threshold. The proposed cut-off resulted in less than $2 \%$ of the acquired aggregates (in the granular fraction) falling below this threshold making it suitable for monitoring purposes. The segmented granules image is then stored, coding the granules entirely within the image limits as 1 , the granules cut-off by the image borders as 0.5 , and the background as 0 . The main steps of QIA methodology for the granules identification can be found in the supplementary material.

With respect to the QIA routine for the flocs identification, following an initial background correction, a histogram equalization and a low pass filter are next applied to enhance the aggregates borders. Flocs segmentation is then achieved using a thresholding algorithm, followed by debris elimination considering a size based morphological operation. The minimum equivalent diameter for a floc to not be considered debris was set to $9 \mu \mathrm{m}$, taking also into account that smaller structures are likely to be composed by a number of bacterial cells hardly composing a full floccular structure. As for the granules, the segmented flocs image is then stored, coding the flocs as well.

The QIA based morphological characterization routine was next performed on the resulting images of the identification step and allowed for the determination of each granule and floc equivalent diameter (calculated from the projected area), length, width, convexity, eccentricity, and robustness parameters used in this analysis. Further information regarding the parameters' calculation can be found in (Amaral, 2003).

\subsection{Decision trees}

A decision tree (DT) feeds an input data matrix to a series of consecutive queries to predict a given output (group allocation) vector (Breiman et al., 1984). The size of the tree (branches number) should be carefully selected to avoid overfitting the output data, which can be accomplished by pruning using k-fold cross-validation (Krzywinksi and Altman, 2017).

In the current work, DT was performed for the ensemble CONT, E2, EE2, and SMX experiments regarding the sample representativeness parameters for the monitoring of the AGS morphology and structure in the presence of PhAC. The main objective of the employed DT focused on assessing the sample representativeness parameters adequateness for the proposed monitoring purposes. Matlab 7.3 (The Mathworks, Inc. Natick) was used for DT calculations.

\subsection{Runs test}

A statistical runs test (Bradley, 1968) was applied to the granules QIA, data obtained for each day of the different experiments, in order to determine the corresponding $z$-value. An $\alpha$-value of 0.05 (95\% confidence) was used, and the obtained $z$-values were compared with the Critical $z$-value (1.96) for that $\alpha$-value. It can be assumed that the data exhibits random behavior for a $z$-value below 1.96, exhibiting nonrandom behavior otherwise. For that purpose, a runstest was used in Matlab 7.3 (The Mathworks, Inc. Natick) for the number of up or down runs on the sequence of observations of the granules QIA parameters to test the hypothesis that the values appear in random order.

\section{Results and discussion}

\subsection{Compliance of the sample volumes for the AGS characterization}

First, an evaluation of the employed aliquot volumes adequateness was performed, regarding the representativeness of the monitored number of granules, for the assessment of the size, morphology, and structure of the AGS. In accordance, the evolution of the granular TSS contents in the reactor, during the different experiments, is presented in Fig. 1.

For the experiments with a granular fraction TSS under $15 \mathrm{~g} \mathrm{~L}^{-1}$ (CONT and E2), a $35 \mathrm{~mL}$ aliquot was used, whereas when the TSS was above $15 \mathrm{~g} \mathrm{~L}^{-1}$ (EE2 and SMX) a $10 \mathrm{~mL}$ aliquot was employed. The reduced volume employed for the experiments presenting larger granular TSS was due to the fact that, for higher biomass contents, the $35 \mathrm{~mL}$ aliquots lead to the acquisition of an excessive number of granules, significantly incrementing the time needed for image acquisition.

First, the sequence of observations of the granules QIA parameters was tested (up and down runs test) for the hypothesis that the values appear in random order. The obtained results confirmed that, with the exception of 16 out of $234(6.8 \%)$ datasets regarding the parameters used in this analysis (and 3 out of the 39 for the diameter), a completely random behavior was observed ( $z$-value below 1.96). Next, the averages and standard deviation values of the selected parameters for sample representativeness (equivalent diameter, length, width, convexity, eccentricity, and robustness) were determined throughout an increasing number of monitored granules (unitary steps) and compared with the respective average and standard deviation values for the entire granules number and each studied sample. A variation 


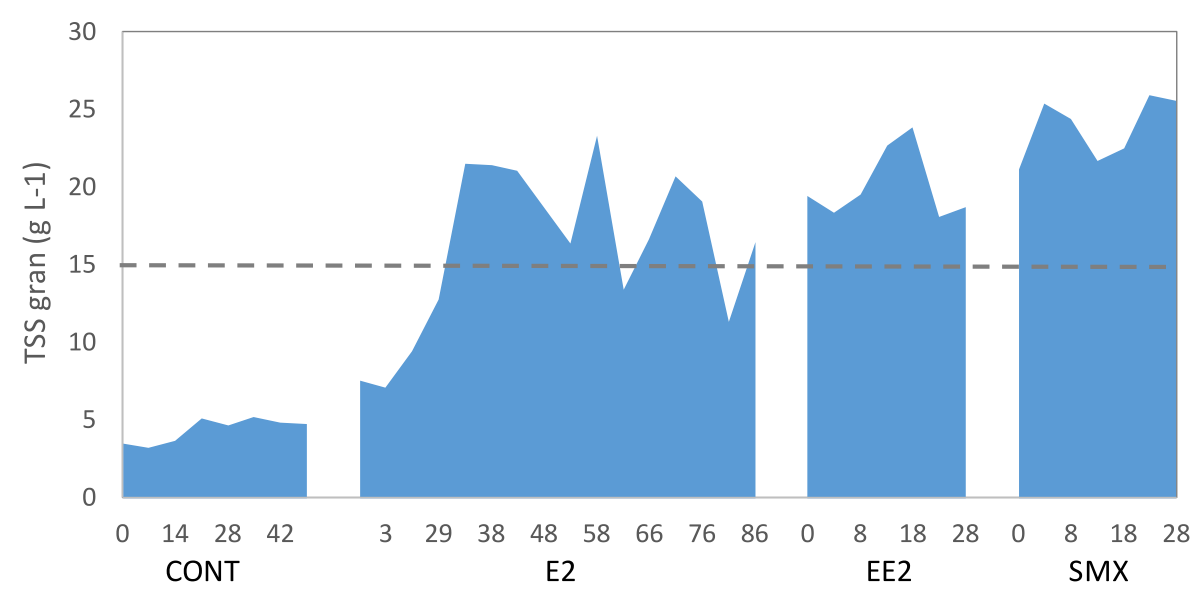

Fig. 1. Granular TSS for each studied sample.

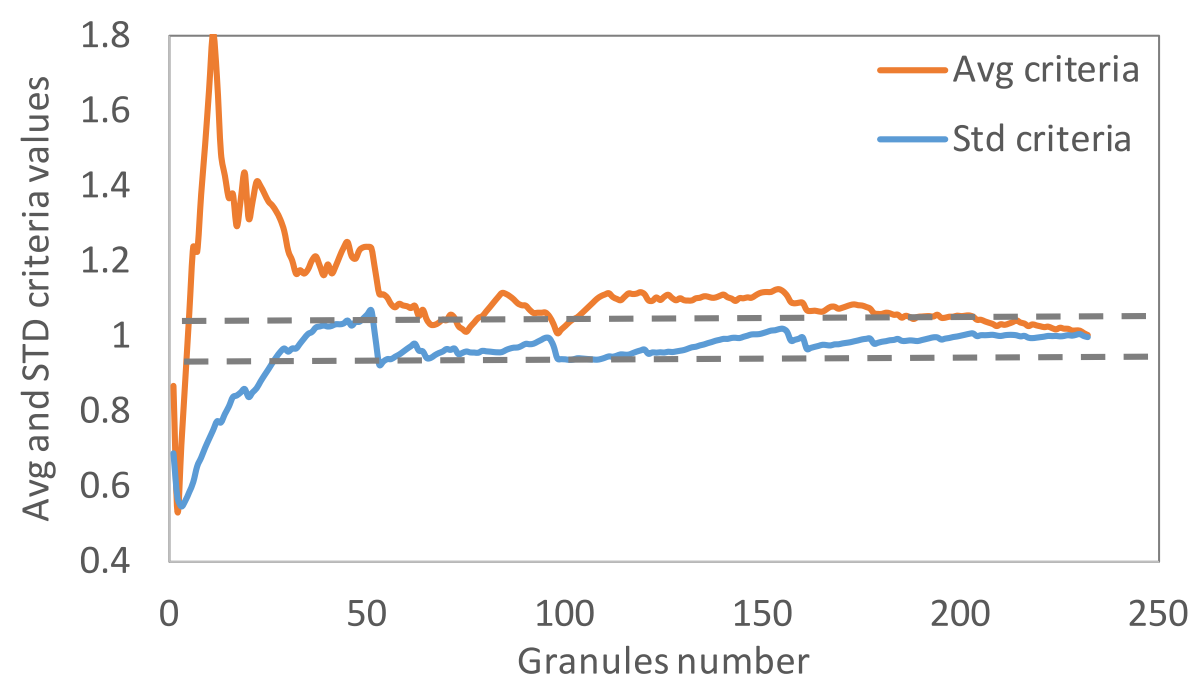

Fig. 2. Average and standard deviations criterion values behavior, for the granules diameter, with the granules number increase, for the first monitored day of the EE2 experiment.

lower, or equal, to $5 \%$ in the average and standard deviations (regarding the entire number of granules) was considered as the goal for the aliquot sample representativeness. As an example, the behavior of the average and standard deviations criterion values for the granules diameter, throughout the increasing number of included granules of the EE2 experiment, is presented for the first monitored day in Fig. 2.

Thereafter, the minimum number of granules that allowed for the average value and standard deviation value criterion to drop below 5\% (0.05 difference) regarding the entire granules number was determined for all experiments. In accordance, the number of monitored granules, and the minimum representative number of granules according to the average and standard deviation criterion, is presented in Fig. 3a), and the complying interval data is presented in Fig. 3b).

Analyzing Fig. 3a) it can be inferred that, according to the average and standard deviation criterion, the employed aliquot volume allowed for a representative sample for all cases and regarding the entire studied parameters. For the CONT and E2 experiments ( $35 \mathrm{~mL}$ aliquot), a variation of less than $5 \%$ in the average and standard deviation values, regarding the entire number of granules, was obtained for an average of 570 granules (with a complying interval averaging 150 granules). That is, the number of acquired granules was, on average, 150 granules higher than the minimum number needed to be representative. Regarding the EE2 and SMX experiments ( $10 \mathrm{~mL}$ aliquot), the necessary number of granules averaged 273 granules (with a complying interval averaging 55 granules).

Taking the above into consideration, it can be inferred that the methodology used to select the granular fraction aliquot volumes, for all cases, was found to be adequate for the QIA assessment of AGS under the employed conditions. Furthermore, it could also be established that the standard deviation criterion was more stringent needing, on average, $18.0 \%$ more granules to be complied than the average criterion. 

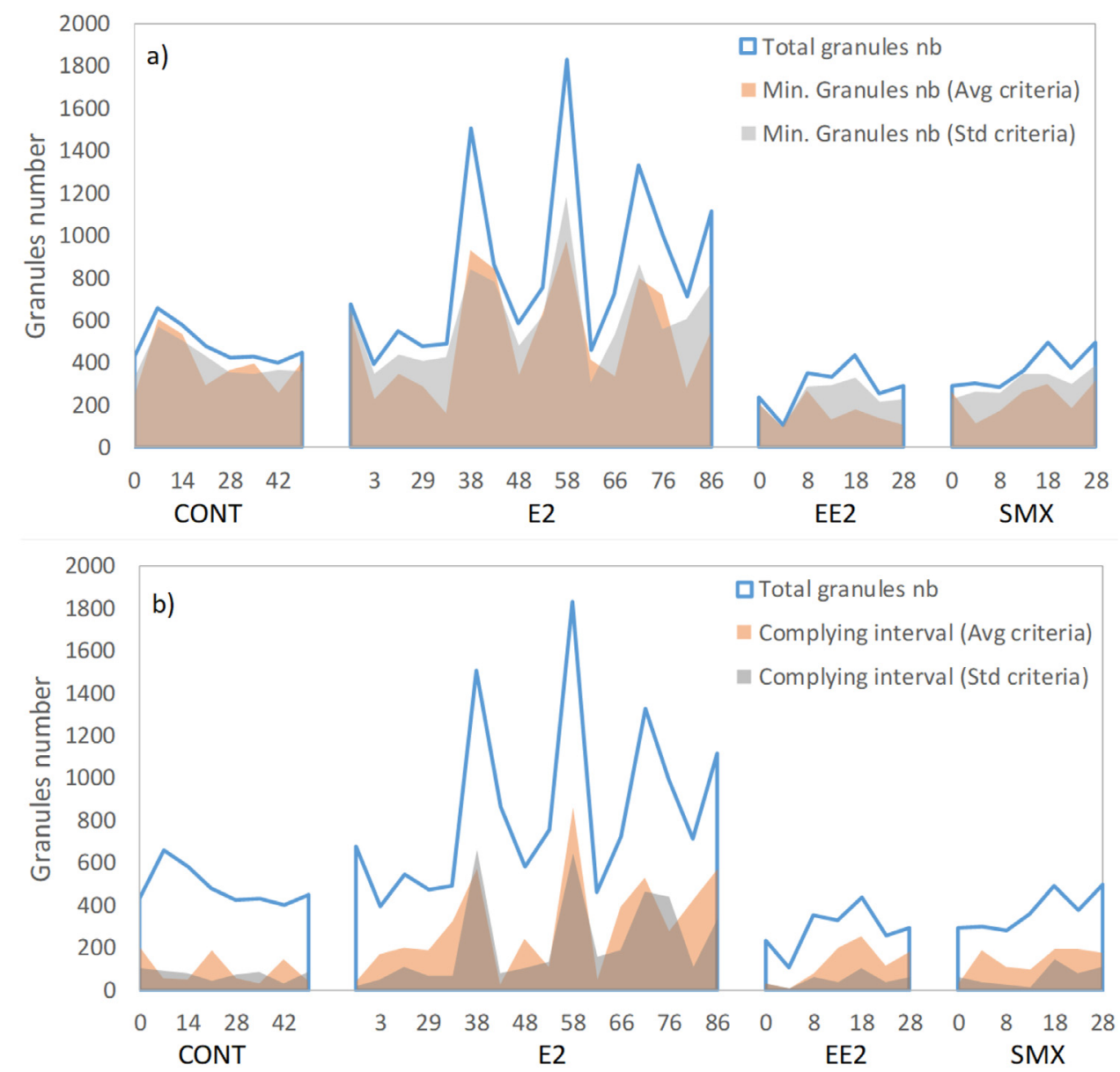

Fig. 3. Total monitored granules and (a) minimum representative number (nb) of granules; (b) complying intervals, according to the average and standard deviation criterion.

With respect to the floccular biomass fraction, the employed methodology has already been proven representative in previous studies (Mesquita et al., 2010a,b).

\subsection{Granular and suspended (floccular) fractions sieving}

With the objective of determining the effectiveness of the sieving (500 $\mu \mathrm{m}$ mesh) biomass separation, the size distribution of the granular and suspended (floccular) fractions was studied. For that purpose, the aggregates above and below $250 \mu \mathrm{m}$ in equivalent diameter (simultaneously the studied largest floccular fraction and smallest granular fraction) of the granular and floccular fractions were compared and are presented in Fig. 4. The floccular biomass fraction (passing through the $500 \mu \mathrm{m}$ sieve) overwhelmingly presented aggregates below $250 \mu \mathrm{m}$ in diameter. On the other hand, with respect to the granular fraction, in some cases a significant percentage $(15.5 \% \pm 9.9 \%)$ consisted on aggregates with an equivalent diameter below $250 \mu \mathrm{m}$ trapped in the sieve. As a result, when considering the granular fraction, as well as for the floccular fraction, the granules separation into size classes is paramount for further enlightenment.

Taking the above into consideration, the granular biomass characterization was, thereafter, performed in three different size classes, namely: below $250 \mu \mathrm{m}$ (in equivalent diameter) - G1; ranging from 250 to $2500 \mu \mathrm{m}$ - G2; and above $2500 \mu \mathrm{m}$ - G3. Concurrently, also for the floccular biomass it has been already proven that its stratification in size classes is of major importance towards their characterization (Mesquita et al., 2011). In accordance, three different size classes were also employed: below $25 \mu \mathrm{m}$ (in equivalent diameter) - F1; ranging from 25 to $250 \mu \mathrm{m}$ - F2; and above $250 \mu \mathrm{m}-\mathrm{F} 3$.

A stratification analysis (Fig. 5), regarding the smallest size class $(<250 \mu \mathrm{m})$ of the granules collected in the $500 \mu \mathrm{m}$ sieve (granular biomass fraction), subjected to the $175 \mu \mathrm{m}$ (debris) cut-off value, allowed to establish a predominance of the $175-200 \mu \mathrm{m}$ class for all experiments, followed by the $200-225 \mu \mathrm{m}$. On the other hand, for most experiments, the percentage of aggregates retained in the sieve below $175 \mu \mathrm{m}$ was negligible $(<2 \%)$. From this analysis, it seems clear a large distribution of size ranges (even quite below $250 \mu \mathrm{m}$ ) for the aggregates quantified as granules (trapped by the employed sieve). 


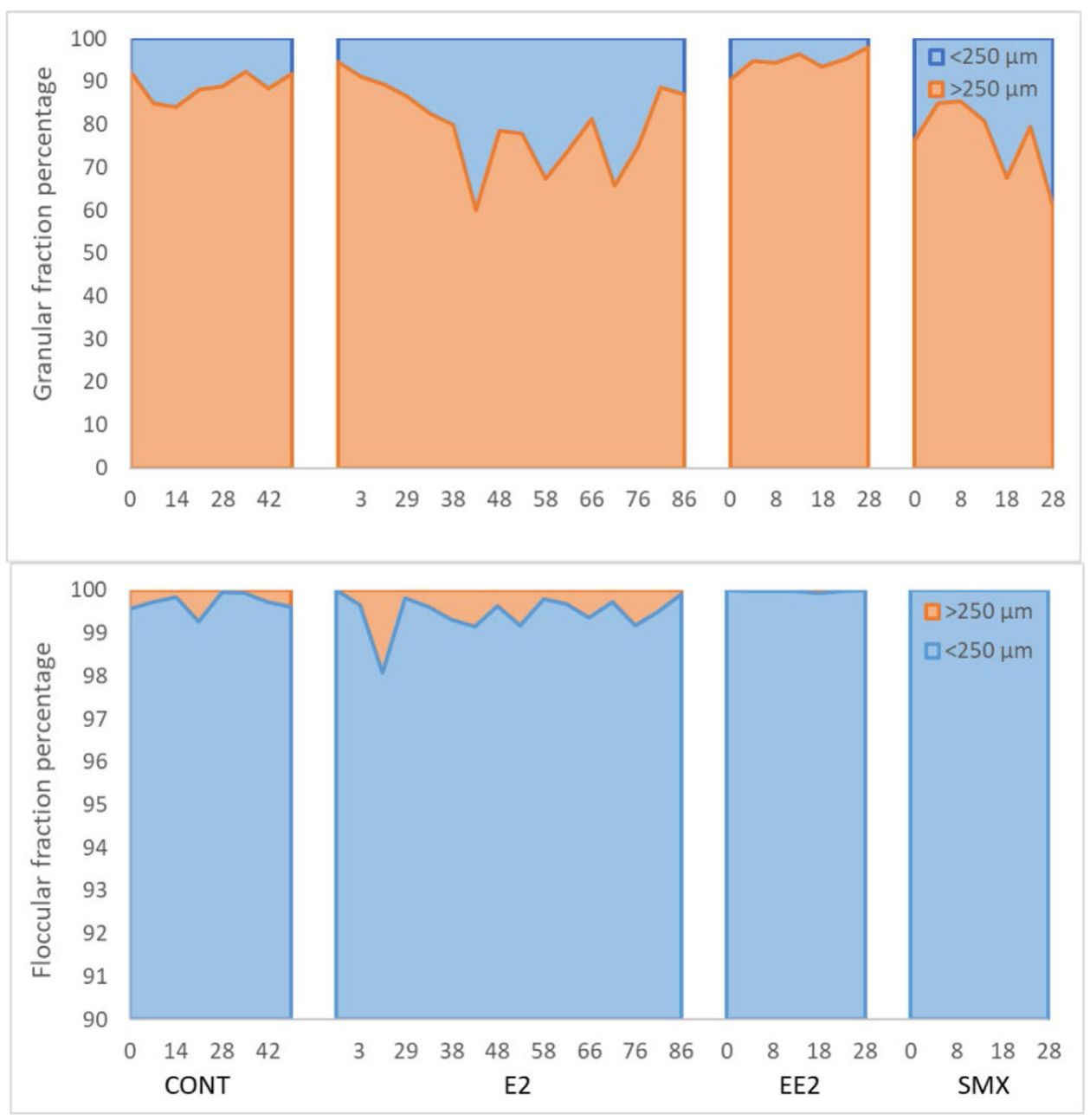

Fig. 4. Aggregates percentage above and below $250 \mu \mathrm{m}$ in equivalent diameter for the granular and floccular fractions.

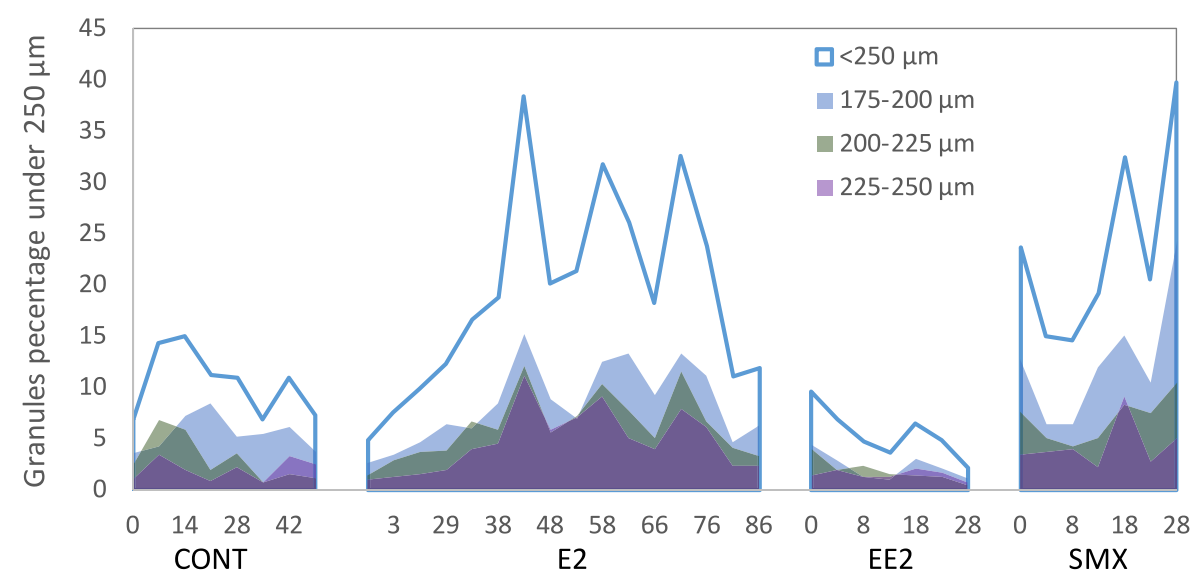

Fig. 5. Stratification analysis presenting the percentage of the aggregates (quantified as granules) below $250 \mu \mathrm{m}$ collected in the $500 \mu \mathrm{m}$ sieve.

Addressing the continuity analysis, upon determining the flocs distribution within the same range (175 to $250 \mu \mathrm{m}$ ), and comparing with the one obtained for the granules, it results clear a similitude between both fractions. In fact, the 175 to $200 \mu \mathrm{m}$ size class was predominant for both aggregates (Table 1) ( $47.3 \%$ to $54.8 \%$ for the ensemble), followed by the 200 to 
Table 1

Aggregates number percentage with respect to the size ranges for the granular and floccular fractions within $175-250 \mu \mathrm{m}$.

\begin{tabular}{lllll}
\hline Fraction & & \multicolumn{3}{l}{ Aggregates number percentage } \\
\cline { 3 - 5 } & & $175-200 \mu \mathrm{m}$ & $200-225 \mu \mathrm{m}$ & $225-250 \mu \mathrm{m}$ \\
\hline \multirow{4}{*}{ Granules } & CONT & 52.5 & 28.6 & 18.9 \\
& E2 & 43.5 & 32.1 & 24.4 \\
& EE2 & 40.5 & 33.2 & 26.3 \\
& SMX & 52.7 & 29.1 & 18.2 \\
& All & & & \\
\multirow{4}{*}{ Flocs } & 47.3 & 30.7 & 18.0 \\
& CONT & 50.6 & 31.4 & 12.7 \\
& E2 & 59.2 & 28.2 & 12.6 \\
& EE2 & 55.2 & 32.2 & 11.8 \\
\hline & SMX & 54.4 & 33.8 & 13.8 \\
\hline
\end{tabular}

${ }^{\mathrm{a}}$ Encompasses all the CONT, E2, EE2 and SMX aggregates within the corresponding size range.

$225 \mu \mathrm{m}$ size class (30.7\% to 31.4\%) and finally by the 225 to $250 \mu \mathrm{m}$ size class (13.8\% to $22.0 \%$ ). Considering the similitude of the three studied size fractions in the lower bound of the granular biomass with the same size fractions of the floccular biomass, this suggests a good agreement between the two acquisition methodologies (flocs and granules), ensuring a continuity in the aggregates determined by them, which reinforces the adequateness of the combined methodology regarding the monitoring purposes.

\subsection{Image acquisition triplicates analysis}

For both granular and floccular fractions, triplicates were acquired and processed by QIA. The obtained results of these triplicates were, thereafter, used to determine the average and standard deviation for each experiment sample. The validation of this approach was performed taking first into account the aggregates diameter parameter and later expanded to the most representative parameters. Accordingly, the average diameters and standard deviations for all experiments and size classes are presented in Fig. 6 . Analyzing the obtained results, it could be established that the standard deviations, with a few exceptions (day 38 of the E2 experiment and day 18 of the SMX experiment, due to the large flocs - F3 low presence, and day 7 of the CONT experiment and days 13 to 28 of the EE2 experiment, due to the low small granules - G1 range), do not encompass, nor significantly affect the validity of the variation found in the parameters.

When normalized by the variation range of the diameters average (for each experiment and size class, represented in Fig. 7) the standard deviation represented, on average, solely $16.6 \%$ of the diameters range variation for the CONT, $11.7 \%$ for the E2, $15.8 \%$ for the EE2 and $15.5 \%$ for the SMX experiments, resulting in a $14.3 \%$ average overall. Furthermore, both the granular and floccular biomass were for the most part in accordance, in average terms, with this behavior, attaining $12.1 \%$ for the flocs and $16.5 \%$ for the granules. With respect to the different studied size classes, the smaller granules (G1) followed by the larger floccular (F3), and granular (G3) aggregates presented the larger standard deviation $(24.3 \%, 19.5 \%$, and $16.4 \%$, respectively) on the aggregates diameter, due to the following main factors: $i$ ) the size range within the smaller granules $(175-250 \mu \mathrm{m})$ is, comparably, the tighter range of all classes; ii) the larger aggregates are not bounded by an upper limit, with the exception of the image size itself; and iii) the aggregates number of the larger aggregates, within each aggregates type, is the lowest, thus leading to more discrepancies. On the opposite, and given their high numbers, the smaller F1 flocs presented the lowest standard deviation (7.2\%).

With respect to the remaining studied parameters, presented in Table 2, the behavior of each parameter standard deviation (\%), regarding the experiments range, followed the established by the diameter parameter. The average values, regarding the ensemble size ranges were somewhat similar for the diameter (14.3\%), length (13.7\%), width (14.4\%), convexity (12.1\%), eccentricity (16.0\%), and robustness (12.2\%). Analyzing in terms of the size fractions, once again the F3 fraction presented the larger values, regarding the ensemble, within the flocs (ranging from $14.8 \%$ in convexity to $20.7 \%$ in width) and the F1 fraction the lowest values (ranging from 5.8\% in robustness to $11.5 \%$ in length). On the other hand, both the granules G1 and G3 fractions presented similar larger results, ranging from $16.5 \%$ to $24.3 \%$ (respectively robustness and diameter in G1) and $10.4 \%$ to $27.1 \%$ (respectively convexity and eccentricity in G3), regarding the ensemble.

In conclusion, it seems feasible to infer that the obtained ensemble average values truly represent the samples real values, within the accuracy ranges presented above. Indeed, in most cases, variations on average of less than $15 \%$ of the studied parameter range, within the experiment, can be obtained.

\subsection{Proof of concept}

In order to evaluate the adequateness of the sample representativeness parameters for the monitoring of the AGS size, morphology and structure in the presence of PhAC, a DT analysis was performed (Fig. 8) and, out of the 38 employed samples in this analysis, a total of $36(94.7 \%)$ were successfully discriminated in the corresponding group. As a result, it could be concluded that the selected parameters allowed to discriminate well between the AGS resulting from the studied PhAC (E2, EE2, and SMX) experiments, as well as regarding the mature granules (CONT) experiment. 

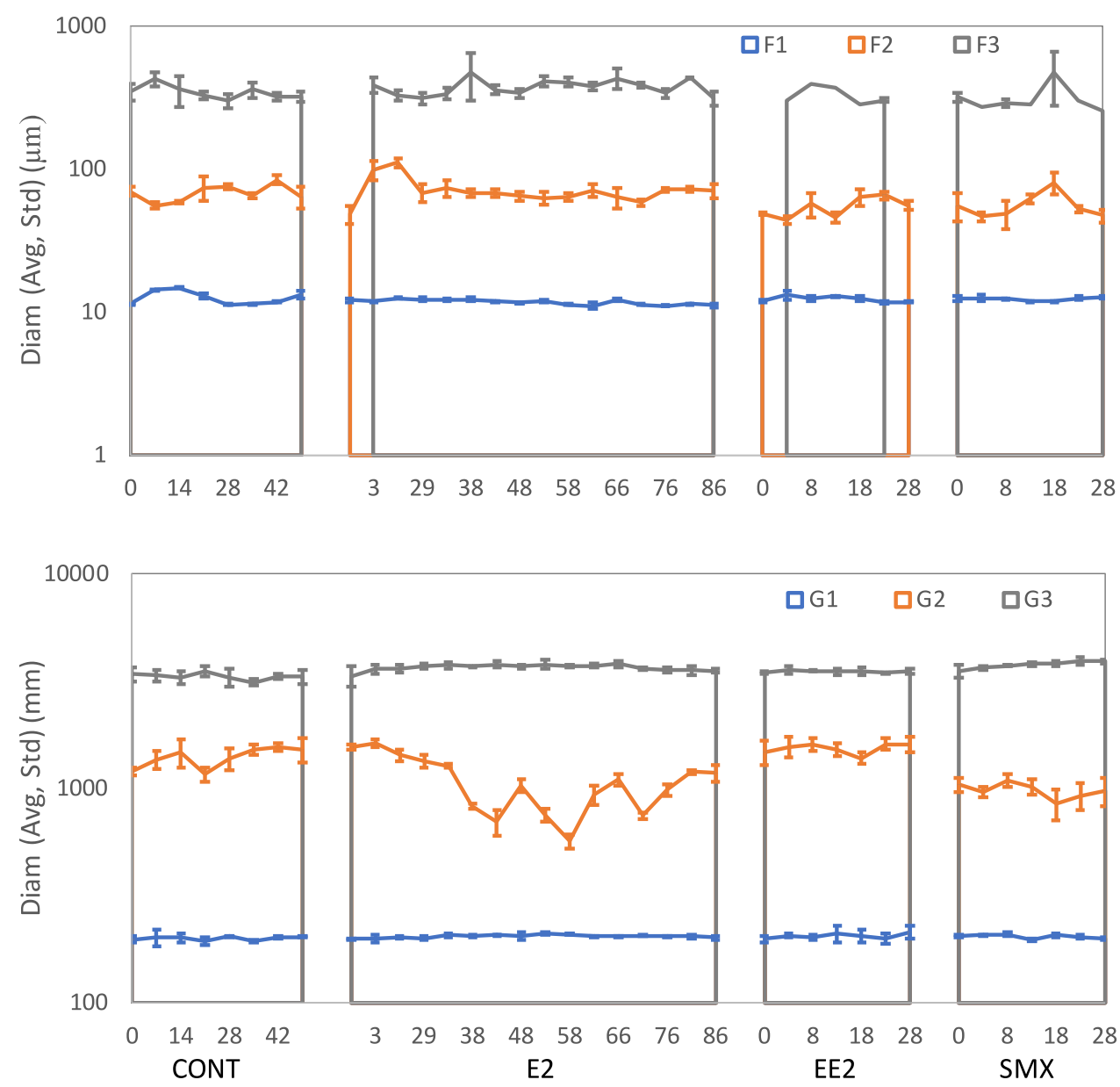

Fig. 6. Average diameter and standard deviation of the granular and floccular fractions.

\section{Conclusions}

In this work the adequateness of a QIA based methodology, including the establishment of the sample volume, the granular and floccular fractions sieving process, the fractions size cut-off and the image acquisition method was evaluated, considering the AGS size, structure, and morphology in synthetic wastewaters containing E2, EE2, and SMX. For that purpose, an approach based on a 5\% variation criterion for a sample average and standard deviation value was employed. The results proved that the employed aliquot volumes, based on the TSS of the granular fraction, were found adequate for the intended purpose. Furthermore, the performance of the sieving process (500 $\mu \mathrm{m}$ sieve), in successfully separating the biomass granular and suspended (floccular) fractions, was also assessed. As a result, a lower bound cut-off granules size of $175 \mu \mathrm{m}$ was established for the employed methodology. Next, a comparison between the 175-250 $\mu \mathrm{m}$ overlapping size range on the floccular and granular biomass proved the continuity of the performed methodology. Since the samples image acquisition was performed in triplicate, a final analysis on the obtained average and standard deviation values was employed. This analysis allowed showing that the experiments variations for the studied parameters could be adequately monitored using triplicates. Furthermore, as a proof of concept, it could be concluded that the selected parameters allowed to discriminate well between the studied PhAC experiments.

Finally, taking into consideration that the proposed methodology has been conducted for lab scale biomass samples, further optimization studies should be considered for the application in pilot and full scale AGS reactors. Consequently, it is expected that, in the future, QIA, and particularly microscopy based AGS survey, could be performed in a regular basis in full scale WWTP, contributing to timely decisions in operation and improving the process control. To that effect, further studies should be performed to evaluate the impact of other variables, such as the employed magnification, in the obtained parameters. 

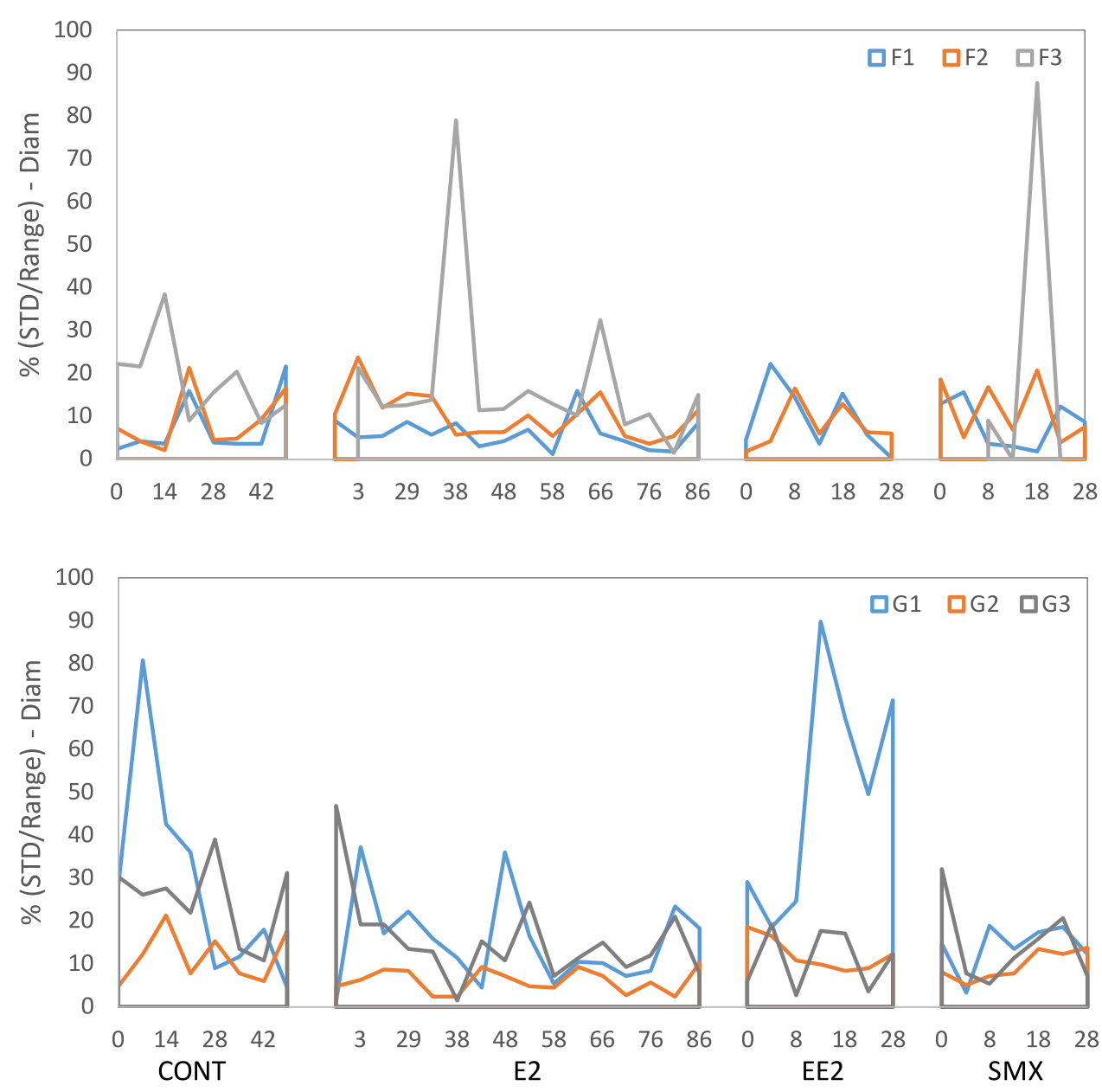

Fig. 7. Standard deviation percentage with respect to the size ranges for the granular and floccular fractions diameter.

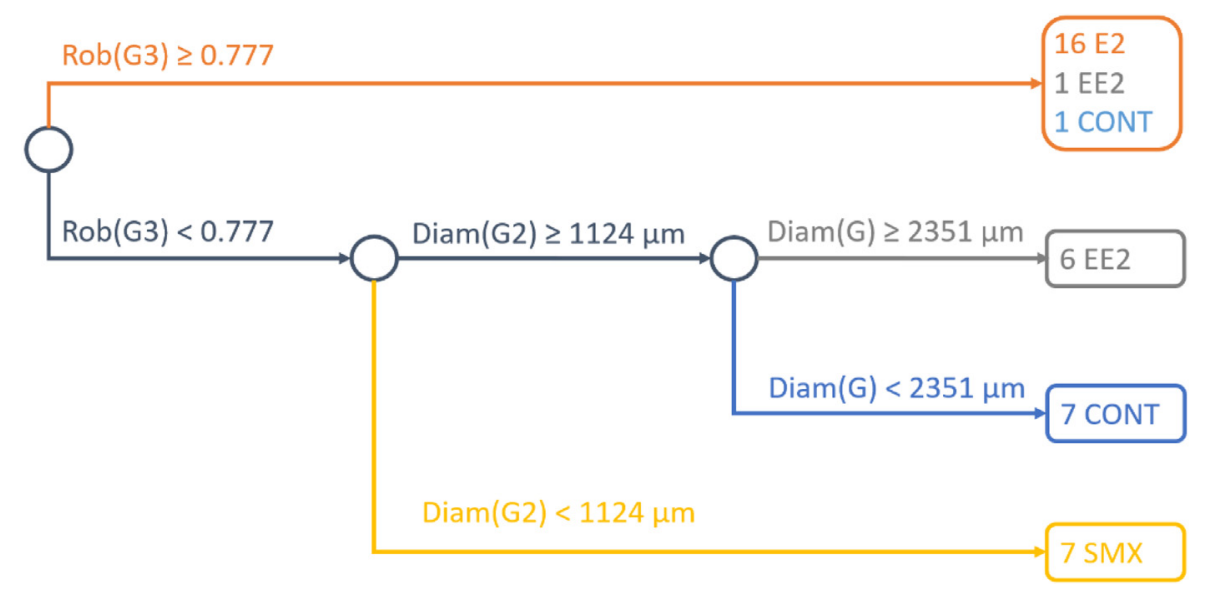

Fig. 8. Results of the performed DT with the sample representativeness parameters (Diam - diameter; Rob. - robustness). 
Table 2

Standard deviation percentage with respect to the size ranges for the granular and floccular fractions and main parameters.

\begin{tabular}{|c|c|c|c|c|c|c|c|}
\hline \multirow[t]{2}{*}{ Parameter } & \multirow[t]{2}{*}{ Fraction } & \multicolumn{6}{|c|}{ Standard deviation percentage } \\
\hline & & $\overline{\mathrm{F} 1}$ & F2 & F3 & G1 & G2 & G3 \\
\hline \multirow{6}{*}{ Diameter } & CONT & 7.2 & 8.6 & 18.4 & 11.9 & 11.5 & 25.0 \\
\hline & E2 & 5.9 & 10.0 & 17.8 & 6.3 & 5.9 & 15.3 \\
\hline & EE2 & 9.3 & 7.5 & 4.9 & 20.6 & 12.0 & 11.2 \\
\hline & SMX & 8.1 & 11.3 & 35.7 & 14.0 & 9.6 & 14.3 \\
\hline & All $^{\mathrm{a}}$ & 7.2 & 9.5 & 19.5 & 24.3 & 8.9 & 16.4 \\
\hline & Ensemble $^{b}$ & 14.3 & & & & & \\
\hline \multirow{6}{*}{ Length } & CONT & 7.9 & 9.4 & 12.7 & 20.8 & 12.8 & 22.8 \\
\hline & E2 & 13.8 & 9.9 & 19.1 & 7.9 & 5.6 & 15.4 \\
\hline & EE2 & 10.4 & 8.3 & 1.5 & 30.2 & 12.1 & 15.1 \\
\hline & SMX & 11.6 & 12.4 & 21.8 & 14.2 & 8.2 & 16.6 \\
\hline & All $^{\mathrm{a}}$ & 11.5 & 10.0 & 16.9 & 17.6 & 8.8 & 17.1 \\
\hline & Ensemble $^{\mathrm{b}}$ & 13.7 & & & & & \\
\hline \multirow{6}{*}{ Width } & CONT & 5.6 & 8.6 & 18.9 & 38.9 & 11.6 & 28.2 \\
\hline & E2 & 6.6 & 10.3 & 18.6 & 10.5 & 6.1 & 17.3 \\
\hline & EE2 & 10.5 & 7.9 & 3.5 & 32.0 & 12.6 & 10.7 \\
\hline & SMX & 8.4 & 11.2 & 42.2 & 14.6 & 9.8 & 13.7 \\
\hline & All $^{\mathrm{a}}$ & 7.5 & 9.6 & 20.7 & 21.9 & 9.1 & 17.7 \\
\hline & Ensemble $^{b}$ & 14.4 & & & & & \\
\hline \multirow{6}{*}{ Convexity } & CONT & 4.8 & 6.1 & 18.2 & 26.9 & 8.1 & 11.6 \\
\hline & E2 & 3.0 & 8.2 & 11.2 & 11.6 & 6.6 & 8.4 \\
\hline & EE2 & 20.1 & 14.3 & 29.2 & 31.2 & 5.1 & 9.7 \\
\hline & SMX & 21.3 & 12.9 & 18.2 & 18.7 & 15.7 & 14.4 \\
\hline & $\mathrm{All}^{\mathrm{a}}$ & 9.9 & 9.7 & 14.8 & 19.7 & 8.3 & 10.4 \\
\hline & Ensemble $^{b}$ & 12.1 & & & & & \\
\hline \multirow{6}{*}{ Eccentricity } & CONT & 7.3 & 6.6 & 15.2 & 41.9 & 7.1 & 42.1 \\
\hline & E2 & 6.3 & 10.9 & 17.0 & 13.3 & 7.6 & 22.5 \\
\hline & EE2 & 7.7 & 17.1 & 0.1 & 33.6 & 14.8 & 28.4 \\
\hline & SMX & 6.8 & 17.1 & 17.9 & 18.9 & 12.9 & 19.3 \\
\hline & All $^{\mathrm{a}}$ & 6.8 & 12.3 & 16.0 & 24.1 & 9.8 & 27.1 \\
\hline & Ensemble $^{b}$ & 16.0 & & & & & \\
\hline \multirow{6}{*}{ Robustness } & CONT & 5.5 & 6.1 & 24.4 & 24.6 & 8.7 & 16.0 \\
\hline & E2 & 3.1 & 9.9 & 10.5 & 9.0 & 5.2 & 13.5 \\
\hline & EE2 & 8.6 & 10.9 & 0.4 & 25.9 & 7.0 & 18.3 \\
\hline & SMX & 9.4 & 11.0 & 54.0 & 14.8 & 12.6 & 13.1 \\
\hline & $\mathrm{All}^{\mathrm{a}}$ & 5.8 & 9.5 & 19.1 & 16.5 & 7.6 & 14.8 \\
\hline & Ensemble $^{\mathrm{b}}$ & 12.2 & & & & & \\
\hline
\end{tabular}

aEncompasses all the CONT, E2, EE2 and SMX aggregates within the corresponding size range.

${ }^{\mathrm{b}}$ Encompasses all the CONT, E2, EE2 and SMX aggregates and all size ranges.

\section{CRediT authorship contribution statement}

Cristiano Leal: Investigation, Writing - original draft. Angeles Val del Río: Investigation, Writing - review \& editing. Eugénio C. Ferreira: Funding acquisition, Writing - review \& editing. Daniela Mesquita: Supervision, Writing - review \& editing. António L. Amaral: Supervision, Writing - review \& editing.

\section{Declaration of competing interest}

The authors declare that they have no known competing financial interests or personal relationships that could have appeared to influence the work reported in this paper.

\section{Acknowledgments}

The authors wish to thank the company Águas do Tejo Atlântico for supplying the aerobic granular sludge. The authors also thank the Portuguese Foundation for Science and Technology (FCT) under the scope of the strategic funding of UID/BIO/04469 unit and the project AGeNT - PTDC/BTA-BTA/31264/2017 (POCI-01-0145-FEDER-031264). Cristiano Leal is recipient of a fellowship supported by a doctoral advanced training (call NORTE-69-2015-15) funded by the European Social Fund under the scope of Norte2020 - Programa Operacional Regional do Norte. A. Val del Rio is supported by Xunta de Galicia, Spain (ED418B 2017/075) and program lacobus (2018/2019). 


\section{Appendix A. Supplementary data}

Supplementary material related to this article can be found online at https://doi.org/10.1016/j.eti.2021.101639.

\section{References}

Amaral, A., 2003. Image Analysis in Biotechnological Processes: Applications to Wastewater Treatment. University of Minho.

APHA, 1998. Standard Methods for the Examination of Water and Wastewater, twentieth ed. American Public Health Association, American Water Works Association and Water Environmental Federation, Washington DC.

Aqeel, H., Weissbrodt, D., Cerruti, M., Wolfaardt, G.M., Wilén, B.-M., Liss, S.N., 2019. Drivers of bioaggregation from flocs to biofilms and granular sludge. Environ. Sci. Water Res. Technol. 5, 20172. http://dx.doi.org/10.1039/c9ew00450e.

Bengtsson, S., de Blois, M., Wilén, B.M., Gustavsson, D., 2019. A comparison of aerobic granular sludge with conventional and compact biological treatment technologies. Environ. Technol. 40 (21), 2769-2778. http://dx.doi.org/10.1080/09593330.2018.1452985.

Bradley, J.V., 1968. Distribution-Free Statistical Tests. Prentice-Hall, Englewood Cliffs, NJ.

Breiman, L., Friedman, J.H., Olshen, R.A., Stone, C.J., 1984. Classification and Regression Trees. Chapman and Hall/CRC, Boca Raton FL.

Cheng, Y., Xuan, X., Zhang, L., Zhao, J., Long, B., 2018. Storage of aerobic granular sludge embedded in agar and its reactivation by real wastewater J. Water Health 16, 958-969. http://dx.doi.org/10.2166/wh.2018.163.

Costa, J.C., Alves, M.M., Ferreira, E.C., 2010. A chemometric tool to monitor high-rate anaerobic granular sludge reactors during load and toxic disturbances. Biochem. Eng. J. 53, 38-43. http://dx.doi.org/10.1016/j.bej.2008.12.006.

De Kreuk, M.K., Heijnen, J.J., van Loosdrecht, M.C.M., 2005. Simultaneous COD, nitrogen, and phosphate removal by aerobic granular sludge. Biotechnol. Bioeng. 90, 761-769. http://dx.doi.org/10.1002/bit.20470.

Jahn, L., Saracevic, E., Svardal, K., Krampe, J., 2019. Anaerobic biodegradation and dewaterability of aerobic granular sludge. J. Chem. Technol Biotechnol. 94, 2908-2916. http://dx.doi.org/10.1002/jctb.6094.

Kirkland, C.M., Krug, J.R., Vergeldt, F.J., van den Berg, L., Velders, A.W., Seymour, J.D., Codd, S.L., van Has, H., de Kreuk, M.K., 2020. Characterizing the structure of aerobic granular sludge using ultra-high field magnetic resonance. Water Sci. Technol. 82 (4), 627-639. http://dx.doi.org/10.2166/ wst.2020.341.

Krzywinksi, M., Altman, N., 2017. Classification and regression trees. Nature Methods 14, 757-758

Leal, C., Mesquita, D.P., Amaral, A.L., Amaral, A.M., Ferreira, E.C., 2020a. Environmental impact and biological removal processes of pharmaceutically active compounds: The particular case of sulfonamides, anticonvulsants and steroid estrogens. Crit. Rev. Environ. Sci. Technol. 50 (7), 698-742. http://dx.doi.org/10.1080/10643389.2019.1642831.

Leal, C., Val del Río, A., Mesquita, D.P., Amaral, A.L., Castro, P.M.L., Ferreira, E.C., 2020b. Sludge volume index and suspended solids estimation of mature aerobic granular sludge by quantitative image analysis and chemometric tools. Sep. Purif. Technol. 234, 116049. http://dx.doi.org/10.1016/ j.seppur.2019.116049.

Li, J., Cai, A., Wang, D., Chen, C., Ni, Y., 2014. Structure analysis of aerobic granule from a sequencing batch reactor for organic matter and ammonia nitrogen removal. Int. J. Environ. Res. Public Health 11, 2427-2436. http://dx.doi.org/10.3390/ijerph110302427.

Li, Z.H., Kuba, T., Kusuda, T., 2006. Selective force and mature phase affect the stability of aerobic granule: An experimental study by applying different removal methods of sludge. Enzyme Microb. Technol. 39, 976-981. http://dx.doi.org/10.1016/j.enzmictec.2006.01.030.

Li, Z., Zhu, Y., Zhang, Ya-li, Zhang, Yu-rong, He, C., 2019. Characterization of aerobic granular sludge of different sizes for nitrogen and phosphorus removal. Eviron. Tech. 40 (27), 3622-3631. http://dx.doi.org/10.1080/09593330.2018.1483971.

Long, B., Xuan, X., Yang, C., Zhang, L., Cheng, Y., Wang, J., 2019. Stability of aerobic granular sludge in a pilot scale sequencing batch reactor enhanced by granular particle size control. Chemosphere 225, 460-469. http://dx.doi.org/10.1016/j.chemosphere.2019.03.048.

Maszenan, A.M., Liu, Y., Jern, W., 2011. Bioremediation of wastewaters with recalcitrant organic compounds and metals by aerobic granules. Biotechnol. Adv 29, 111-123. http://dx.doi.org/10.1016/j.biotechadv.2010.09.004.

Mesquita, D.P., Amaral, A.L., Ferreira, E.C., 2011. Characterization of activated sludge abnormalities by image analysis and chemometric techniques Anal. Chim. Acta 705, 235-242. http://dx.doi.org/10.1016/j.aca.2011.05.050

Mesquita, D.P., Dias, O., Amaral, A.L., Ferreira, E.C., 2010a. A comparison between bright field and phase-contrast image analysis techniques in activated sludge morphological characterization. Microsc. Microanal. 16 (2), 166-174. http://dx.doi.org/10.1017/S1431927609991358.

Mesquita, D.P., Dias, O., Elias, R.A.V., Amaral, A.L., Ferreira, E.C., 2010b. Dilution and magnification effects on image analysis applications in activated sludge characterization. Microsc. Microanal. 16 (5), 561-568. http://dx.doi.org/10.1017/S1431927610093785.

Nancharaiah, Y.V., Kumar, G.K.K., 2017. Aerobic granular sludge technology: mechanisms of granulation and biotechnological applications. Bioresour. Technol. 247, 1128-1143. http://dx.doi.org/10.1016/j.biortech.2017.09.131.

Pronk, M., Bassin, J.P., de reuk, M.K., Kleerebezem, R., van Loosdrecht, M.C.M., 2014. Evaluating the main and side effects of high salinity on aerobic granular sludge. Appl. Microbiol. Biotechnol. 98, 1339-1348. http://dx.doi.org/10.1007/s00253-013-4912-z.

Pronk, M., de Kreuk, M.K., de Bruin, B., Kamminga, P., Kleerebezem, R., van Loosdrecht, M.C.M., 2015. Full scale performance of the aerobic granular sludge process for sewage treatment. Water Res. 84, 207-217. http://dx.doi.org/10.1016/j.watres.2015.07.011.

Ranzinger, F., Matern, M., Layer, M., Guthausen, G., Wagner, M., Derlon, N., Horn, H., 2020. Transport and retention of artificial and real wastewater particles inside a bed of settled aerobic granular sludge assessed applying magnetic resonance imaging. Water Res. X 7 , 100050. http://dx.doi.org/10.1016/j.wroa.2020.100050.

Rusanowska, P., Cydzik-Kwiatkowska, A., Świątczak, P., Wojnowska-Baryła, I., 2019. Changes in extracellular polymeric substances (EPS) content and composition in aerobic granule size-fractions during reactor cycles at different organic loads. Bioresour. Technol. 272, 188-193. http: //dx.doi.org/10.1016/j.biortech.2018.10.022.

Sheng, G.P., Li, A.J., Li, X.Y., Yu, H.Q., 2010. Effects of seed sludge properties and selective biomass discharge on aerobic sludge granulation. Chem. Eng. J. 160, 108-114. http://dx.doi.org/10.1016/j.cej.2010.03.017.

Verawaty, M., Tait, S., Pijuan, M., Yuan, Z., Bond, P.L., 2013. Breakage and growth towards a stable aerobic granule size during the treatment of wastewater. Water Res. 47, 5338-5349. http://dx.doi.org/10.1016/j.watres.2013.06.012.

Zhang, W., Jiang, F., 2019. Membrane fouling in aerobic granular sludge (AGS) -membrane bioreactor (MBR): Effect of AGS size. Water Res. 157, 445-453. http://dx.doi.org/10.1016/j.watres.2018.07.069.

Zhang, C., Zhang, H., Yang, F., 2015. Diameter control and stability maintenance of aerobic granular sludge in an A/O/A SBR. Sep. Purif. Technol. 149, 362-369. http://dx.doi.org/10.1016/j.seppur.2015.06.010.

Zhou, J., Zhang, Z., Zhao, H., Yu, H., Alvarez, P.J.J., Xu, X., 2016. Bioresource technology optimizing granules size distribution for aerobic granular sludge stability : Effect of a novel funnel-shaped internals on hydraulic shear stress. Bioresour. Technol. 216, 562-570. http://dx.doi.org/10.1016/ j.biortech.2016.05.079.

Zhu, L., Yu, Y., Dai, X., Xu, X., Qi, H., 2013. Optimization of selective sludge discharge mode for enhancing the stability of aerobic granular sludge process. Chem. Eng. J. 217, 442-446. http://dx.doi.org/10.1016/j.cej.2012.11.132. 